\title{
Preclinical Evaluation of Chimeric Antigen Receptor-Modified T Cells Specific to Epithelial Cell Adhesion Molecule for Treating Colorectal Cancer
}

\author{
Bing-Lan Zhang, ${ }^{1,2}$ Dan Li, ${ }^{1}$ You-Ling Gong, ${ }^{1,3}$ Yong Huang, ${ }^{1}$ Di-Yuan Oin, \\ Lin Jiang, ${ }^{1}$ Xiao Liang, ${ }^{1}$ Xiao Yang, ${ }^{1}$ Hong-Feng Gou, ${ }^{4}$ Yong-Sheng Wang, ${ }^{1,3}$ \\ Yu-Quan Wei, ${ }^{1 *}$ and Wei Wang ${ }^{1 *}$ \\ ${ }^{1}$ Department of Biotherapy, ${ }^{3}$ Department of Thoracic Oncology, and ${ }^{4}$ Department of Medical Oncology, State Key Laboratory of Biotherapy and Cancer Center, West \\ China Hospital, Sichuan University, and Collaborative Innovation Center for Biotherapy, Chengdu, P.R. China; ${ }^{2}$ Department of Gastroenterology, the First Affiliated \\ Hospital of Chongqing Medical University, Chongqing, P.R. China.
}

Chimeric antigen receptor-modified $\mathrm{T}$ cells (CAR-T cells) have emerged as a promising cancer immunotherapy for solid tumors. Epithelial cell adhesion molecule (EpCAM) is overexpressed in a variety of tumors and is recognized as a biomarker for circulating tumor cells and cancer stem cells, representing an attractive target for adoptive T-cell immunotherapy. This study generated third-generation CAR-T cells with redirected specificity to EpCAM (EpCAM CAR-T) by lentiviral vector. The study demonstrated that EpCAM CAR-T cells can elicit lytic cytotoxicity to target cells in an EpCAM-dependent manner and secrete cytotoxic cytokines, including interferon gamma and tumor necrosis factor alpha. Furthermore, adoptive transfer of EpCAM CAR-T cells significantly delayed tumor growth and formation in xenograft models. In addition, the safety evaluation showed that CAR-T cells have no systemic toxicity in mice. The data confirmed the antitumor ability and safety of CAR-T cells targeting EpCAM and may provide a new target for CAR-T cell therapies in treating solid tumors.

Keywords: chimeric antigen receptor, EpCAM, cancer immunotherapy, colorectal cancer

\section{INTRODUCTION}

EPITHELIAL CELL ADHESION MOLECULE (EpCAM) is a transmembrane glycoprotein originally discovered on colon carcinomas. ${ }^{1}$ Subsequent research showed it to be overexpressed to varying degrees in most human carcinomas. ${ }^{2}$ It has been shown that EpCAM abrogates E-cadherin-mediated cell-cell adhesion, thereby loosening cell-cell adhesion, promoting cell motility, proliferation, carcinogenesis, and metastasis formation. ${ }^{3}$ Numerous studies have reported that the overexpression of EpCAM is associated with decreased overall survival of patients with many tumors. ${ }^{4-7}$

Recently, EpCAM was identified as a biomarker for circulating tumor cells (CTCs) and cancer stem cells (CSCs). CTCs are the potential precursors of tumor metastasis that actively invaded or have been shed from the primary tumor into the blood circulation. ${ }^{8}$ CTC capture using EpCAM-based gating is feasible for most cancer subtypes, especially for breast cancer. ${ }^{9-12}$ In addition, CSCs, which maintain cancer tissues by sustaining phenotypically diverse cancer cells, are considered as pivotal target for the eradication of cancer. EpCAM is expressed on CSCs from breast, colon, pancreas, and prostate tumors. ${ }^{13-16}$ CSCs have a high level of resistance to chemotherapy and radiotherapy, ${ }^{17}$ which makes EpCAM an even more interesting target for cancer immunotherapy.

EpCAM has been targeted with monoclonal antibodies $(\mathrm{mAb})$ in treating various cancers. Edrecolomab was the first EpCAM-directed mAb

${ }^{*}$ Correspondence: Dr. Wei Wang or Prof. Yu-Quan Wei, State Key Laboratory of Biotherapy/Collaborative Innovation Center of Biotherapy, West China Hospital, Sichuan University, Chengdu 610041,P.R. China.E-mail: weiwang@scu.edu.cn or yqwei@scu.edu.cn 
approved in Germany for the adjuvant treatment of colorectal cancer. ${ }^{18}$ However, the results of subsequent clinical trials for edrecolomab were invalid, and it was subsequently withdrawn from the market. ${ }^{19,20}$ Recent advances in potentiating the antitumor effects of anti-EpCAM mAb rely on creating multifunctional antibodies, including bispecific antibodies and trifunctional antibodies capable of simultaneous binding to different targets on tumor or immune cells. Recently, a trifunctional mAb, Catumaxomab, has been approved by the European Medicines Agency (EMA) for malignant ascites in patients with $\mathrm{EpCAM}^{+}$carcinomas. Clinical trials using anti-EpCAM mAb have met variable success in breast, colon, pancreas, prostate, and ovarian carcinoma. $^{20-24}$ This discrepancy may be attributed to EpCAM variance in expression density and low affinity to the target antigen.

Chimeric antigen receptor-modified T cells (CAR$\mathrm{T}$ cells) have recently formed a part of a broad wave of immunotherapies that are showing promise in early-phase cancer clinical trials. These CAR-T cells modified with a recombinant receptor molecule recognize cell-surface antigens directly and are independent of major histocompatibility complex (MHC) restrictions. Recent reports on the impressive efficacy of CAR-T cells against hematologic malignancies have inspired oncologists to extend their efforts for the treatment of solid tumors.

This study constructed a third-generation CAR recognizing EpCAM and transduced T cells by the lentiviral vector to redirect $\mathrm{T}$ cells with specificity to EpCAM (EpCAM CAR-T). Then, it evaluated whether the EpCAM CAR-T cells could inhibit the growth of solid tumors in vitro and in vivo and the safety of EpCAM CAR-T cells in xenograft mice. Finally, EpCAM CAR-T cells were shown to elicit lytic cytotoxicity to target cells in an EpCAMdependent manner, and adoptive transfer of EpCAM CAR-T cells significantly delayed tumor growth and formation against human colon cancers, without signs of severe adverse effects. This preclinical study of EpCAM CAR-T suggests that adoptive transfer of CAR-T cells targeting EpCAM is safe and efficacious and is a promising therapeutic strategy for treating $\mathrm{EpCAM}^{+}$solid tumors.

\section{METHODS Cell lines}

The human cervical cancer cell line Hela, human non-small cell lung cancer (NSCLC) cell line A549, human breast cancer cell line MDA-MB-231, human colon cancer cell lines HT29 and SW480, and lentivirus packaging cell line HEK 293TD were obtained from the American Type Culture Collection (Manassas, VA). All the cell lines were cultured in Dulbecco's modified Eagle's medium (Invitrogen, Grand Island, NY) supplemented with $10 \%$ heat-inactivated fetal bovine serum.

Human peripheral blood mononuclear cells (PBMCs) were isolated from voluntary donors with their consent. All the experiments were approved by the Ethics Committee of the State Key Laboratory of Biotherapy. After centrifugation on FicollHypaque density gradients (Sigma-Aldrich, St. Louis, MO), PBMCs were cultured in X-VIVO 15 medium (Lonza, Basel, Switzerland) supplemented with $2.5 \%$ human $\mathrm{AB}$ serum, $100 \mathrm{IU} / \mathrm{mL}$ recombinant human interleukin-2 (rhIL-2), and stimulated with anti-CD3 and anti-CD28 magnetic beads (Invitrogen, Carlsbad, CA).

\section{CAR construction}

The anti-EpCAM single-chain variable fragment sequence $(\mathrm{scFv})$ was screened by the authors' laboratory in the early stages. The hinge region of $\mathrm{CD} 8 \alpha$, transmembrane domain of the $\mathrm{CD} 8 \alpha$, and intracellular region $\mathrm{CD} 28,4-1 \mathrm{BB}$ and $\mathrm{CD} 3 \zeta$, simply called 8a28BBZ, were directly synthesized by GenScript. The sequences of $\mathrm{scFv}$ and $8 \mathrm{a} 28 \mathrm{BBZ}$ were spliced using overlapping polymerase chain reaction (PCR) to form the EpCAM-specific CAR. Finally, the EpCAM-specific CAR was inserted into a pWPXLD (Addgene, Cambridge, MA) lentiviral vector using BamHI and EcoRI enzymes. The whole sequence was confirmed by direct sequencing.

\section{Lentivirus production}

Lentiviruses were produced in HEK-293 TD cells using the calcium phosphate method. The pWPXLD-based plasmid and the packaging plasmids psPAX2 and PMD2.0G (Invitrogen) were used at a ratio of 2:2:1. The supernatants containing the lentivirus particles were harvested 48 and $72 \mathrm{~h}$ post transfection, filtered, and concentrated through ultracentrifugation at $70,000 \mathrm{~g}$ for 120 min. Mock lentivirus was produced using an empty pWPXLD lentiviral plasmid. The concentrated lentivirus titers were determined by Lentiviral p24 enzyme-linked immunosorbent assay (ELISA; Clontech, Mountain View, CA) or quantitative reverse transcription PCR. Finally, the concentrated lentivirus was stored at $-80^{\circ} \mathrm{C}$. The average ranges of the titers for the concentrated lentivirus were about $10^{8-9} \mathrm{IFU} / \mathrm{mL}$.

\section{Lentivirus transduction}

The isolated PBMCs described above were activated for 1 day to stimulate $\mathrm{T}$ cells selectively. The 
non-tissue culture six-well plates were coated with $1 \mathrm{~mL}$ RetroNectin $(50 \mu \mathrm{g} / \mathrm{mL})$ in phosphatebuffered saline (PBS) overnight at $4^{\circ} \mathrm{C}$. The next day, the RetroNectin solution was aspirated, and the wells were blocked with $1 \mathrm{~mL}$ PBS solution $+2 \%$ bovine serum albumin for $30 \mathrm{~min}$ at room temperature. The blocking solution was then aspirated, and the wells were washed twice with PBS solution. The concentrated pWPXLD-CAR-encoding lentivirus or mock lentivirus was diluted and added to each RetroNectin-coated well. The six-well plates were centrifuged at $1,000 \mathrm{~g}$ for $2 \mathrm{~h}$ at $32^{\circ} \mathrm{C}$. After centrifugation, activated $\mathrm{T}$ cells were added to each well and incubated at $37^{\circ} \mathrm{C}$ with $5 \% \mathrm{CO}_{2}$ for two consecutive days. Forty-eight hours later, T cells were replaced with new medium, cultured, and expanded for 1-2 weeks before analysis.

\section{Flow cytometry}

The expression levels of EpCAM on tumor cell lines were examined using flow cytometry. Briefly, $10 \mu \mathrm{L}$ phycoerythrin (PE)-conjugated anti-human EpCAM antibody (BioLegend, San Diego, CA) was added to $1 \times 10^{6}$ cells suspended in $100 \mu \mathrm{L}$ PBS. After 30 min of staining at $4^{\circ} \mathrm{C}$, cells were washed three times with PBS and detected using a flow cytometer (FACSCalibur; BD Biosciences, San Jose, CA). For analysis of CAR expression, the modified $\mathrm{T}$ cells were stained with $\mathrm{PE}$-conjugated anti-F(ab)2 (Jackson ImmunoResearch, West Grove, PA). The phenotype of CAR-T cells was evaluated by staining with anti-CD3, anti-CD8, anti-CD4, anti-CD45RO, and anti-CD62L antibodies (BioLegend). Flow cytometry data were acquired with a FACSCalibur flow cytometer (BD Biosciences) and analyzed with FlowJo v7.6.

\section{Cytotoxicity and co-culture assays}

Cytotoxicity of CAR-T cells to target cells was first determined using a standard ${ }^{51} \mathrm{Cr}$ release assay at a different effector cell:target cell ratio $(\mathrm{E}: \mathrm{T}$ ratio $=2.5 / 5 / 10$ ) as described previously. ${ }^{25}$ In addition, CAR-T cells were co-cultured with a panel of $\mathrm{EpCAM}^{+}$or $\mathrm{EpCAM}^{-}$tumor cell lines at a different E:T ratio in 96-well plates, and the supernatants were collected at $24 \mathrm{~h}$. ELISA was used to detect interferon gamma (IFN- $\gamma$ ) and tumor necrosis factor alpha (TNF- $\alpha$ ) secretion (eBioscience, Inc., San Diego, CA).

\section{Mouse xenograft model}

Female NOD/SCID BALB/c mice (all 5-8 weeks of age) were obtained from the Beijing HFK Bioscience Co. Ltd. (Beijing, P.R. China). In vivo experiments were performed in accordance with the animal healthcare regulations of the State Key Laboratory of Biotherapy, Sichuan University. To examine the effect of EpCAM CAR-T cells on tumor formation, $5 \times 10^{6}$ HT29 and SW480 cells were respectively co-inoculated subcutaneously (hind flank) with $2 \times 10^{7}$ EpCAM-specific CAR-modified $\mathrm{T}$ lymphocytes (a mix of transduced and nontransduced cells) or mock $\mathrm{T}$ cells in NOD-SCID BALB/c mice. Only HT29 or SW480 tumor cells were inoculated in the NS group. Five mice per group were used. Once the tumors were palpable, the tumor growth was monitored by caliper measurement every 3 days. Tumor volume was calculated using the following formula: length $\times$ width $^{2} \times 0.52$ ).

\section{Analysis of toxic effects in mice}

All the NOD/SCID BALB/c mice were evaluated for evidence of acute toxic effects induced by CAR-T cell therapy. Animals were sacrificed when the control group was on the verge of death. Blood samples were collected, and serum was separated. All the values in the routine blood test and blood biochemical test were measured at the State Key Laboratory of Biotherapy (Chengdu, P.R. China). After sacrifice, the main visceral tissues in the HT29 and SW480 models were fixed by $4 \%$ polyformaldehyde solution and then processed for paraffin sections. Four-micron sections of the excised visceral tissues were stained with hematoxylin and eosin (H\&E) for morphologic analysis.

\section{Statistical analysis}

All the data are expressed as the mean \pm standard deviation. Statistics analysis was performed using GraphPad Prism v5.0 (GraphPad Software, Inc., San Diego, CA). Statistical analysis for IFN- $\gamma$ and TNF- $\alpha$ secretion was performed using a paired Student's $t$-test. One-way analysis of variance was used to compare the tumor volume curves. $p$-Values of $<0.05$ were considered statistically significant.

\section{RESULTS}

\section{Construction of EpCAM-specific CAR}

A third-generation EpCAM-specific CAR was successfully constructed, which was composed of a signal peptide (Sp) of hIL-2, anti-EpCAM scFv, CD8 $\alpha$-hinge, transmembrane of CD8 $\alpha(\mathrm{CD} 8 \alpha-\mathrm{TM})$, co-stimulatory molecules CD28 and $4-1 \mathrm{BB}$, and human $\mathrm{CD} 3 \zeta$ domain (Fig. $1 \mathrm{~A}$ and $\mathrm{B}$ ). Among these, $\mathrm{scFv}$ is responsible for the binding of tumor antigen. CD $8 \alpha$-hinge mediates the dimerization of CAR. CD8 $\alpha$-TM anchors the CAR on the membrane of the lymphocytes. CD28 and 4-1BB are co-stimulatory molecules that can improve the cytotoxic activity 

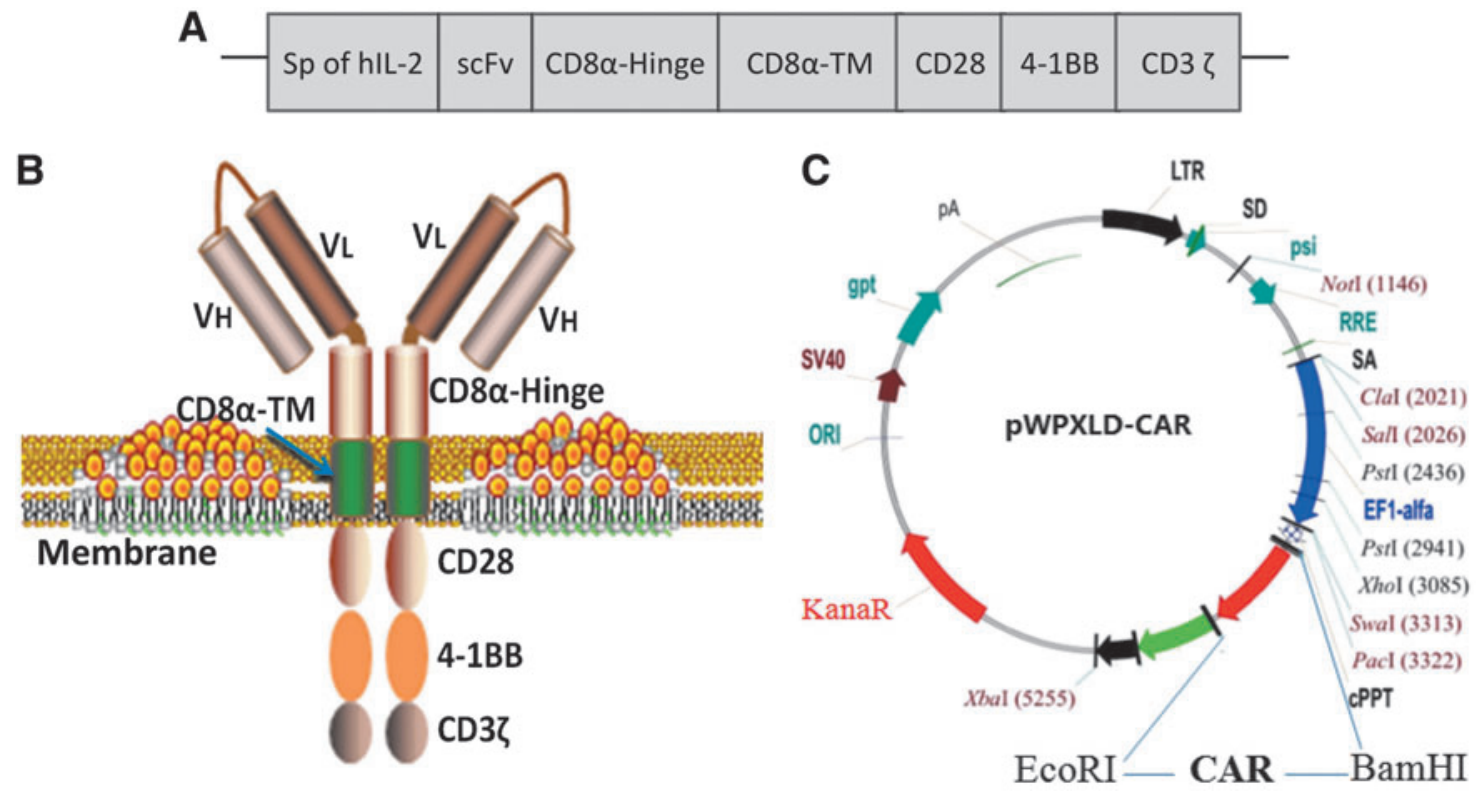

Figure 1. Construction of epithelial cell adhesion molecule (EpCAM)-specific chimeric antigen receptor (CAR). (A and B) Schematic diagram of EpCAMspecific CAR, consisting of signal peptide of human interleukin-2 (Sp of hlL-2), single-chain variable fragment (scFv) targeting to EpCAM, extracellular domain of $\mathrm{CD} 8 \alpha$ (CD $8 \alpha$-hinge), transmembrane of $\operatorname{CD} 8 \alpha(\mathrm{CD} 8 \alpha-\mathrm{TM}), \mathrm{CD} 28$ and 4-1BB costimulatory molecules, and human $\mathrm{CD} 3 \zeta$ domain. (C)This CAR gene was cloned into $\mathrm{BamHI}$ and $\mathrm{EcoRI}$ sites of an EF1-alfa promoter-based lentiviral vector, pWPXLD.

and proliferation capacity of $\mathrm{T}$ cells, and extend the survival time of $\mathrm{T}$ cells. ${ }^{26-28} \mathrm{CD} 3 \zeta$ transmits the signal elicited from the binding of antigen by the scFv. The CAR with EpCAM specificity was inserted into a pWPXLD lentiviral vector using BamHI and EcoRI enzymes (Fig. 1C). The whole sequence was then confirmed by direct sequencing.

\section{Efficient generation of CAR-T cells}

To achieve stable expression of CAR on T cells, first, lentivirus encoding the green fluorescent protein (GFP) was used to explore the conditions of infection. The schematic representation of pWPXLD-GFP is shown in Supplementary Fig. S1A. Human PBMCs were separated and stimulated with anti CD3/CD28 immune magnetic beads for 1 day before being transduced with lentiviruses encoding GFP. Two days later, the transduction efficiency was determined by flow cytometry and monitored under a fluorescent microscope. As shown in Supplementary Fig. 1B, $47.9 \%$ of PBMCs expressed GFP by flow cytometry (multiplicity of infection $[\mathrm{MOI}]=5$ ). Expression of GFP was further confirmed under a fluorescence microscope, indicating that high transfection efficiency was obtained (Supplementary Fig. 1C). As above, human PBMCs were stimulated and transduced with lentiviruses encoding the EpCAM-specific CAR. Two days later, the transduction efficiency was determined by staining with anti-F(ab)2 for flow cytometry and anti-CD3 $\zeta$ antibody for Western blot analysis. As shown in Fig. 2A, 31.8\% of PBMCs expressed the CAR by flow cytometry $(\mathrm{MOI}=10)$. Because a CD3 $\zeta$ sequence was included in the EpCAMspecific CAR (Fig. 1A and B), a protein with an expected molecular weight of $55 \mathrm{kD}$ was detected by Western blot (Fig. 2B). Protein with a weight of $16 \mathrm{kD}$ is endogenous $\mathrm{CD} 3 \zeta$, which could be detected in all the $\mathrm{T}$ lymphocytes. The result revealed that the CAR specific to EpCAM is successfully transduced and expressed on human $\mathrm{T}$ lymphocytes.

Ten days later, the phenotypes of all transduced $\mathrm{T}$ cells were examined by staining with anti-CD3, anti-CD8, and anti-CD4 antibodies. CD3 was expressed by $>95 \%$ of CAR-T cells, of which $70.4 \%$ were $\mathrm{CD}^{+}$and $24.7 \%$ were $\mathrm{CD}^{+}$(Fig. 2C). The result show that the percentage of $\mathrm{CD}^{+} \mathrm{T}$ cells is obvious more than $\mathrm{CD} 4^{+} \mathrm{T}$ cells after amplification, which suggests that $\mathrm{CD}^{+} \mathrm{T}$ cells may have a stronger killing ability. Recently, CD45RO and CD62L were used to identify the type of memory $\mathrm{T}$ cells, which can be divided into three categories: central memory $\mathrm{T}$ cells $\left(\mathrm{T}_{\mathrm{CM}}, \mathrm{CD} 45 \mathrm{RO}^{+} \mathrm{CD} 2 \mathrm{~L}^{+}\right)$, effector memory $\mathrm{T}$ cells $\left(\mathrm{T}_{\mathrm{EM}}, \mathrm{CD} 45 \mathrm{RO}^{+} \mathrm{CD} 6 \mathrm{~L}^{-}\right.$), and native $\mathrm{T}$ cells $\left(\mathrm{T}_{\mathrm{N}}, \mathrm{CD} 45 \mathrm{RO}^{-} \mathrm{CD} 2 \mathrm{~L}^{+}\right) .{ }^{29,30} \mathrm{In}$ the present study, $\mathrm{T}_{\mathrm{CM}}$ accounted for the majority of the CAR-T cells (87.3\%), $\mathrm{T}_{\mathrm{EM}}$ accounted for $7.08 \%$, while $\mathrm{T}_{\mathrm{N}}$ only accounted for $4.81 \%$ (Fig. $2 \mathrm{C}$ ). 


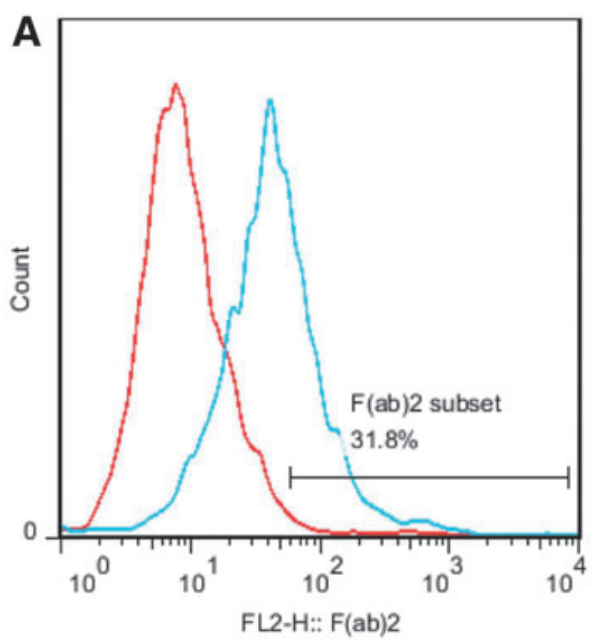

B
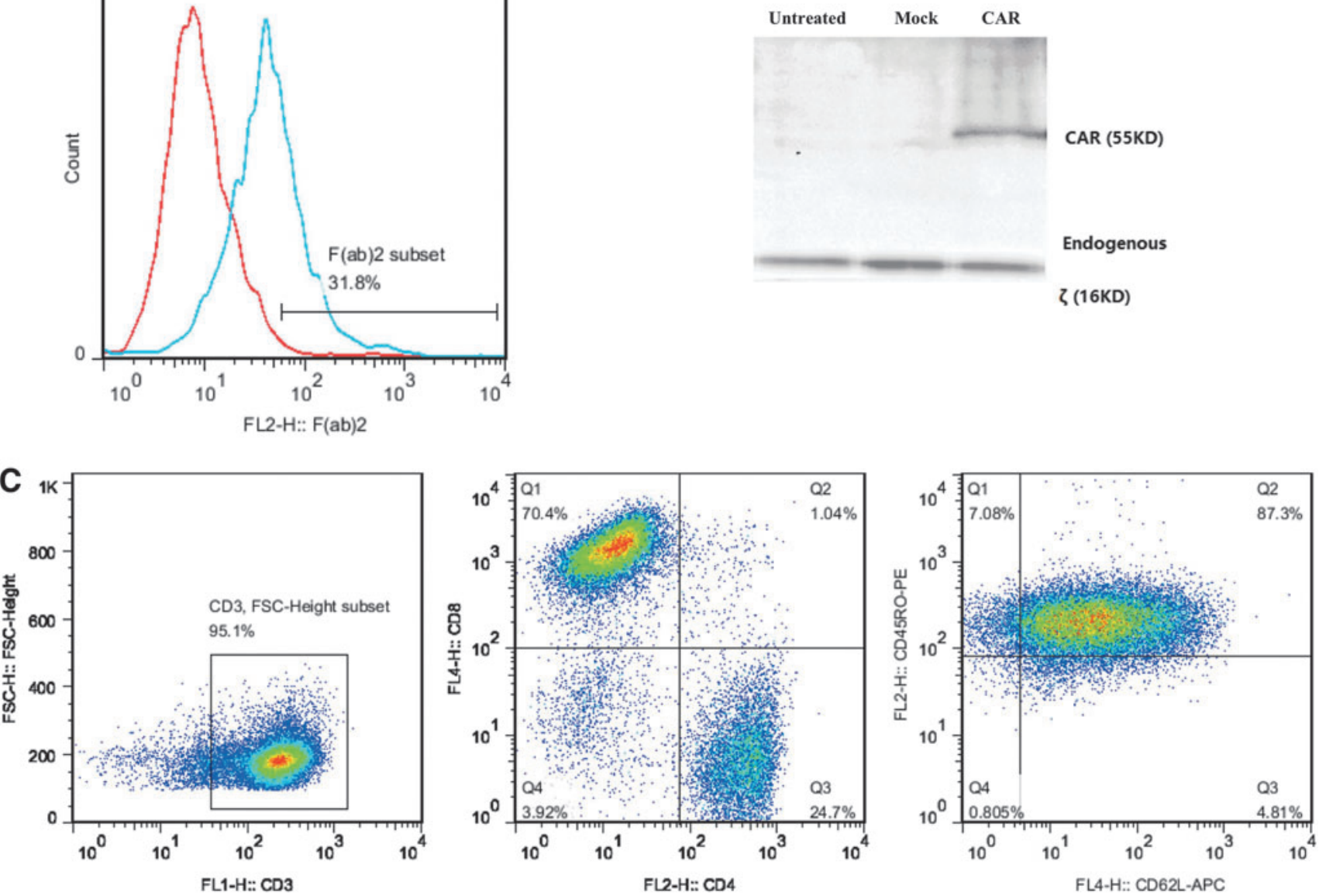

Figure 2. Expression of EpCAM-specific CAR on T lymphocytes and the phenotypes of CAR-T cells. (A) Flow cytometry analysis of the EpCAM-specific CAR expression on the modified T cells. F(ab)2 subset is the proportion of T cells expressing CAR. T lymphocytes transfected with lentivirus encoding pWPXLD lentiviral vector were used as a negative control. The red line indicates the negative control group while the blue line indicates the CAR group. (B) Detection of EpCAM-specific CAR expression by Western blot. (C) Representative expression of CD3, CD8, CD4, CD45R0, and CD62L markers on EpCAM-specific CAR-T cells by flow cytometry. Numbers represent percentages of cells per quadrant.

\section{EpCAM CAR-T cells elicit specific cytotoxicity to target cells expressing the EpCAM antigen}

EpCAM expression in various tumor cell lines was independently validated by flow cytometry. EpCAM is highly expressed in the human colon cancer cell lines HT29 and SW480, and is moderately expressed in the human NSCLC cell line A549 and the human breast cancer cell line MDAMB231, while it is not expressed in the human cervical cancer cell line Hela (Fig. 3A).

To investigate whether the EpCAM CAR-T cells were capable of specifically recognizing tumor cells, CAR-T cells were co-incubated with a panel of Ep$\mathrm{CAM}^{+}$or $\mathrm{EpCAM}^{-}$tumor cell lines $(\mathrm{E}: \mathrm{T}$ ratio $=5)$. Twenty hours later, the amount of secreted effector cytokines including IFN- $\gamma$ and TNF- $\alpha$ were determined by ELISA. The result showed that CAR-T cells can specifically recognize $\mathrm{EpCAM}^{+}$tumor cell lines, including A549, MDA-MB-231, SW480, and HT29, and secrete high-dose IFN- $\gamma$ (Fig. 3B) and
TNF- $\alpha$ (Fig. 3C) compared to MOCK T cells (NoCAR). For the EpCAM ${ }^{-}$tumor cell line Hela, there was no significant difference in the level of IFN $-\gamma$ and TNF- $\alpha$ between the EpCAM CAR-T group and NoCAR T group (Fig. 3B and C).

Next, the cytotoxicity of CAR-T cells was evaluated using a ${ }^{51} \mathrm{Cr}$ release assay. As shown in Fig. 4A-E, T lymphocytes redirected against EpCAM were demonstrated to elicit lytic cytotoxicity to target cells in an EpCAM-dependent manner. None of the T cells killed the EpCAM ${ }^{-}$Hela cells. Thus, the result further confirmed that EpCAMspecific CAR-T cells can display specific and efficient targeting of $\mathrm{EpCAM}^{+}$tumor cells.

\section{EpCAM-specific CAR-T cells delay the tumor growth of mice with subcutaneous EpCAM-expressing colon tumors}

To investigate the ability of the EpCAM-specific CAR-T cells to control tumor growth in vivo, two 

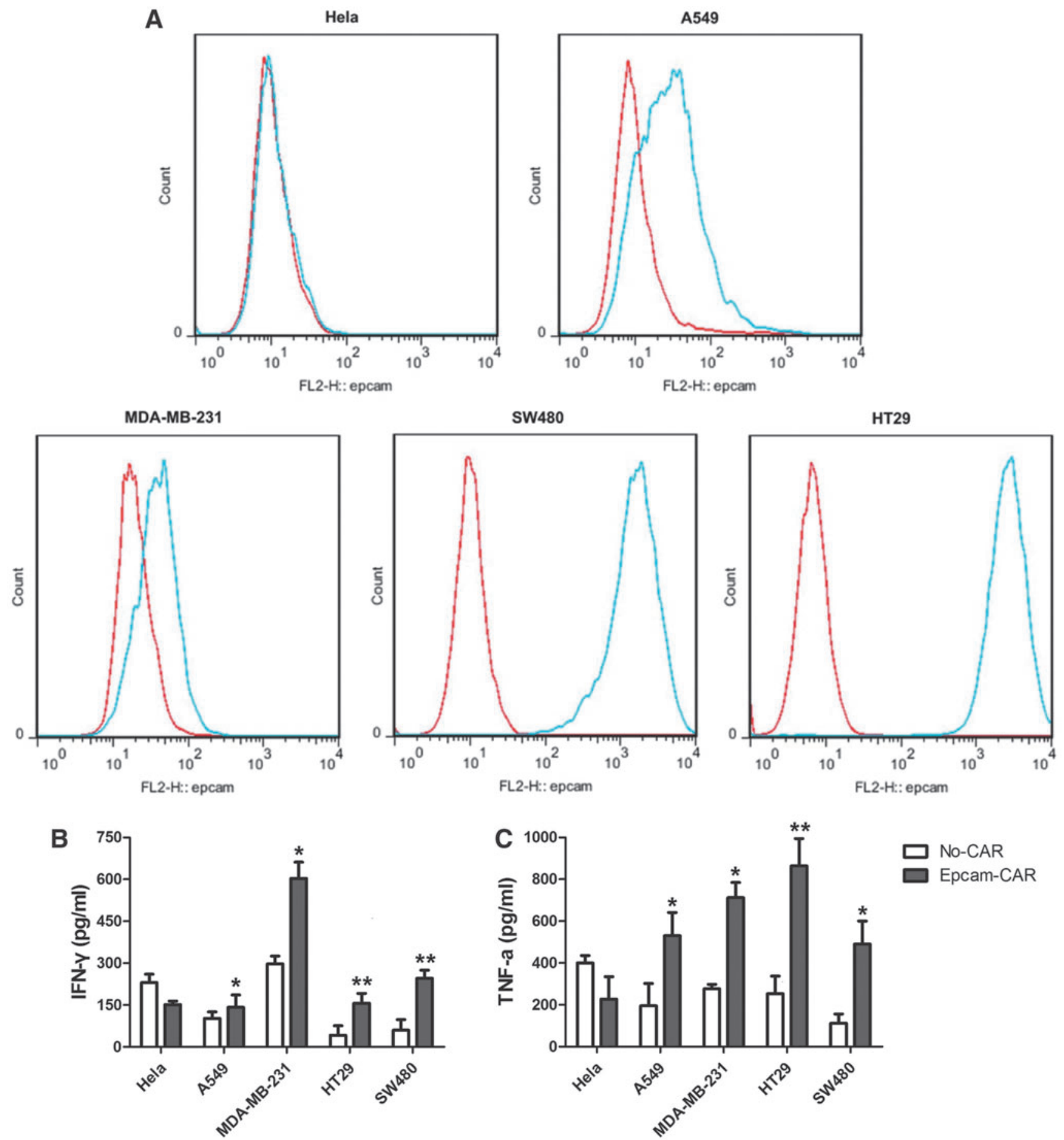

Figure 3. Specific interferon gamma (IFN- $\gamma$ ) and tumor necrosis factor alpha (TNF- $\alpha$ ) release of T lymphocytes transduced with EpCAM-specific CAR. (A) The expression of EpCAM on a variety of tumor cells was detected by flow cytometry analysis. The red line indicates the isotype control while the blue line indicates the tumor cells stained with anti-EpCAM antibody. (B) CAR-T cells and control T cells were co-incubated with a panel of EpCAM ${ }^{+}$or EpCAM ${ }^{-}$tumor cell lines (E:T ratio $\left.=5: 1\right)$. Twenty hours after co-culture, a specific enzyme-linked immunosorbent assay was used to analyze the supernatant for IFN- $\gamma$ cytokine-release. Results are presented as the mean \pm standard deviation $(S D)$. (C) The detection of TNF- $\alpha$ in the same culture supernatant. Results are presented as the mean $\pm S D$. ${ }^{*} p<0.05$; ${ }^{* *} p<0.01$.

colon tumor cell lines, HT29 and SW480, with high expression levels of EpCAM were selected as xenograft tumor models. EpCAM CAR-T cells or mock T cells were co-inoculated with HT29 or SW480 tumor cells in NOD/SCID BALB/c mice, and HT29 or SW480 tumor cells alone were inoc- ulated in the NS group. Compared to control T lymphocytes, the adoptive transfer of EpCAMspecific CAR-modified T lymphocytes significantly delayed tumor formation and growth in HT29 and SW480 xenograft models (Fig. 5). By day 26, HT29 colon tumors reached an average volume of 


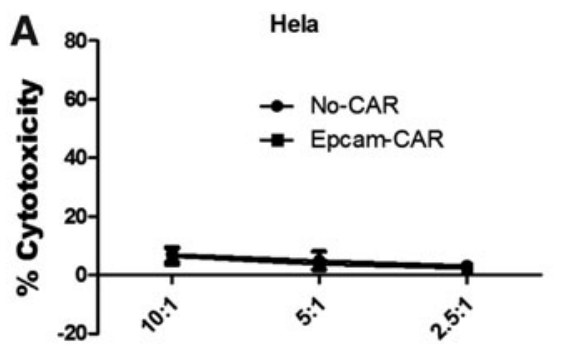

E:T ratio

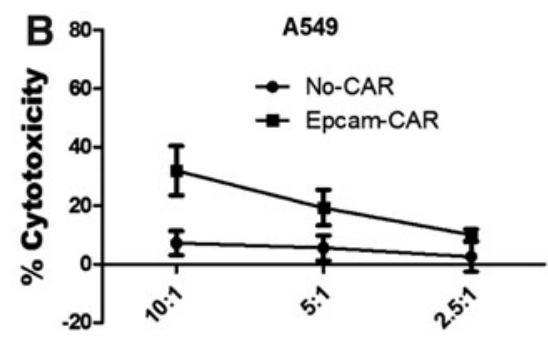

E:T ratio

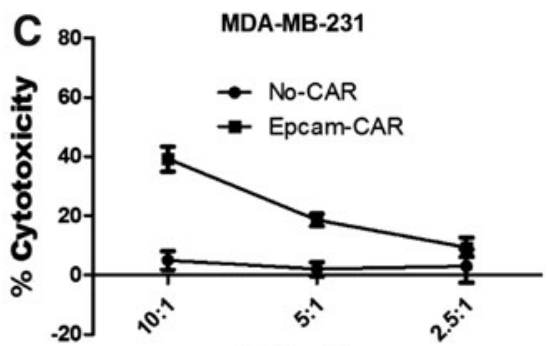

E:T ratio

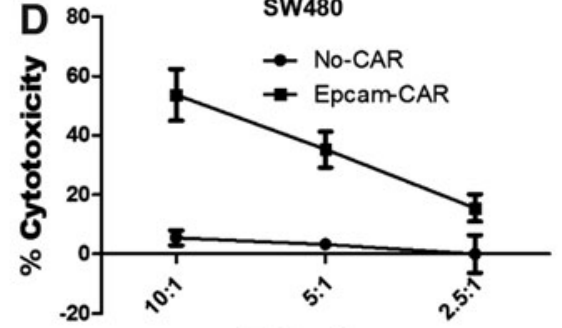

E:T ratio

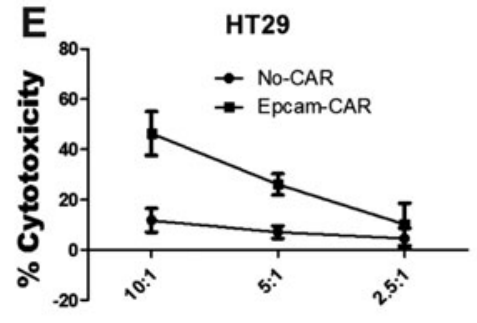

E:T ratio

Figure 4. Cytotoxic function of EpCAM-specific CAR-T cells against tumor cells. A standard ${ }^{51} \mathrm{Cr}$ release assay was performed to assess the cytotoxicity. Target cells used were EpCAM ${ }^{-}$tumor cell Hela (A) and EpCAM ${ }^{+}$tumor cell A549 (B), MDA-MB-231 (C), SW480 (D), and HT29 (E). CAR-T cells redirected against EpCAM were demonstrated to elicit lytic cytotoxicity to target cells in an EpCAM-dependent manner. No CAR-T cells were transfected by the lentivirus coding vector pWPXLD. E:T ratio, the ratio of effector cells to target cells.
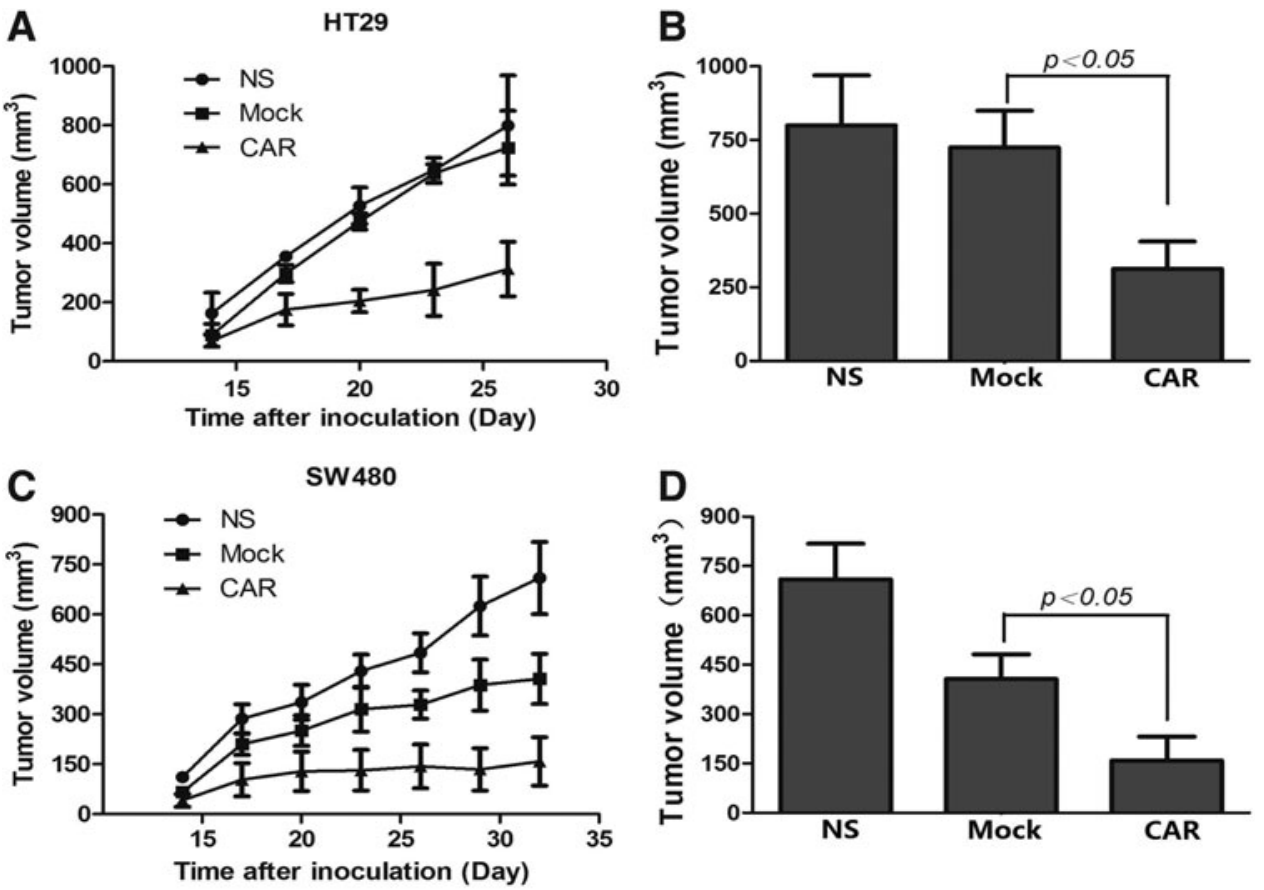

Figure 5. EpCAM-specific CAR-T cells control tumor growth in vivo. T lymphoctytes transfected with lentivirus encoding EpCAM-specific CAR (group CAR-T) or pWPXLD lentiviral vector (mock group) were co-inoculated with HT29 or SW480 tumor cells in mice at an E:T ratio of 4:1. Only tumor cells were inoculated in the NS group. ( $\mathbf{A}$ and $\mathbf{C}$ ) Tumor volumes were measured every 3 days and are shown as the mean $\pm S D$ on the tumor growth curves. (B and $\mathbf{D}$ ) All mice were sacrificed, and the mean tumor volume was calculated. 
$313 \pm 92 \mathrm{~mm}^{3}$ in mice receiving EpCAM CAR-T cells compared to $725 \pm 125 \mathrm{~mm}^{3}$ in mice receiving mock T cells and $800 \pm 170 \mathrm{~mm}^{3}$ in mice in the NS group ( $p<0.05$; Fig. 5A and B). In the SW480 tumor model, the average tumor volume on day 32 for the CAR-T group was $159 \pm 73 \mathrm{~mm}^{3}$ compared to the average tumor volume of $407 \pm 75 \mathrm{~mm}^{3}$ observed in the mock group and $710 \pm 108 \mathrm{~mm}^{3}$ observed in NS mice $(p<0.05$; Fig. $5 \mathrm{C}$ and $\mathrm{D})$.

\section{Safety evaluation of EpCAM CAR-T cells in mice}

The potential toxic effects in the mice were evaluated in preclinical experiments. There were no graft-versus-host reactions such as a rash or diarrhea during the whole treatment process in any of the mice. Blood samples were collected from the mice after they were sacrificed, and all the main routine blood tests and biochemical tests were undertaken. There was no significant difference between the EpCAM CAR-T group and the control groups (Figs. $6 \mathrm{~A}$ and $\mathrm{B}$ and $7 \mathrm{~A}$ and $\mathrm{B}$ ). Compared to mice from the NS and mock groups, no toxic pathologic changes in the heart, liver, spleen, lungs, or kidneys were detected by microscopic examination after H\&E staining in the CART group (Figs. 6C and 7C). Collectively, these data suggested that treatment with EpCAM CAR-T cells is safe in vivo in mice.

\section{DISCUSSION}

The impressive therapeutic benefits of CAR-T cell therapy have been widely demonstrated in patients suffering with hematologic malignancies. ${ }^{31}$ For solid tumors, it is critical to find an ideal tumor target antigen. EpCAM is highly expressed in various types of cancers and is involved in pro-
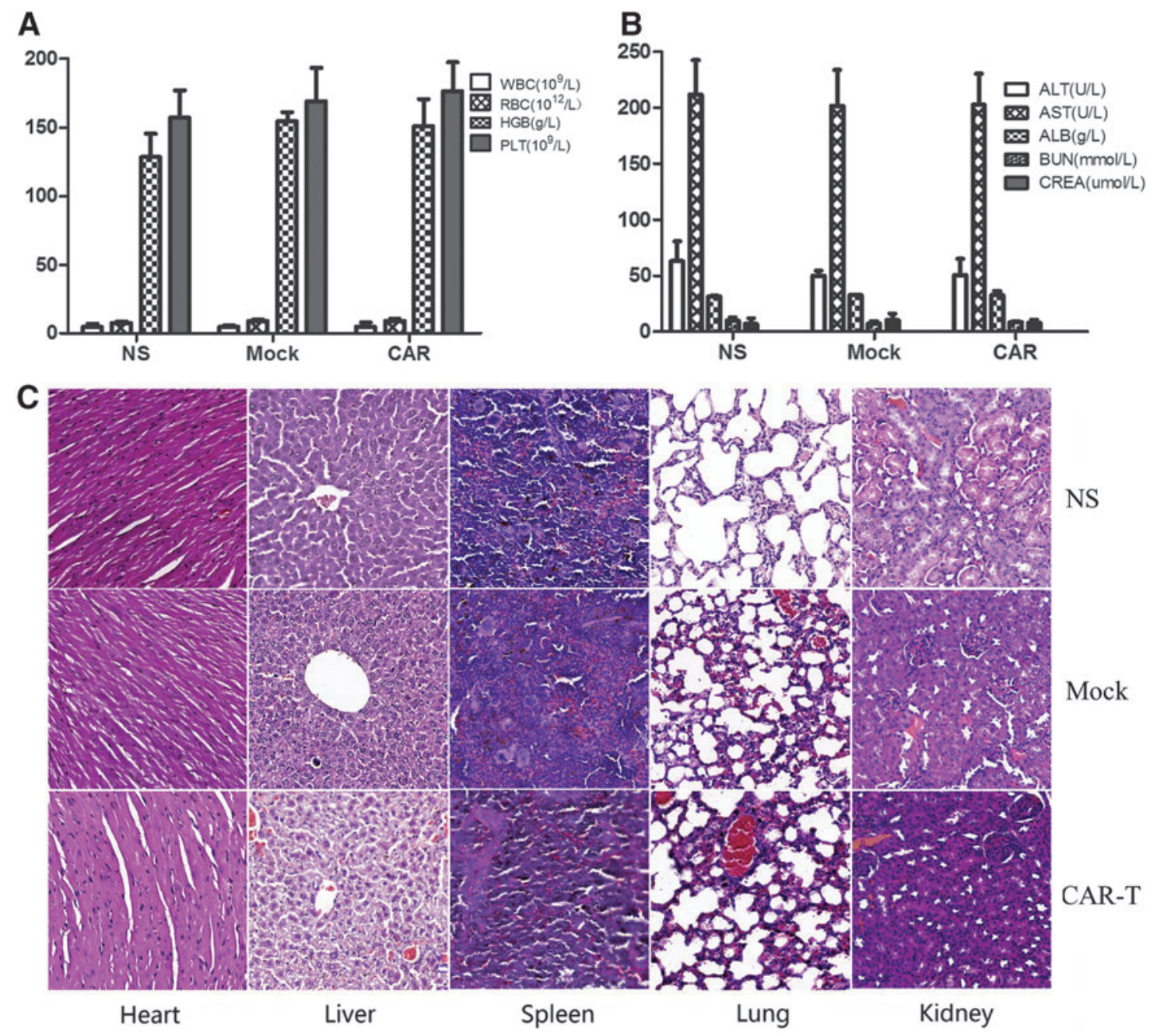

Figure 6. Systemic toxicity evaluation of CAR-modified T cells in the HT29 tumor model. (A) Routine blood test results. (B) Blood biochemical test results. (C) Hematoxylin and eosin (H\&E) staining of visceral tissues, including the heart, liver, spleen, lung, and kidney in the HT29 model. 

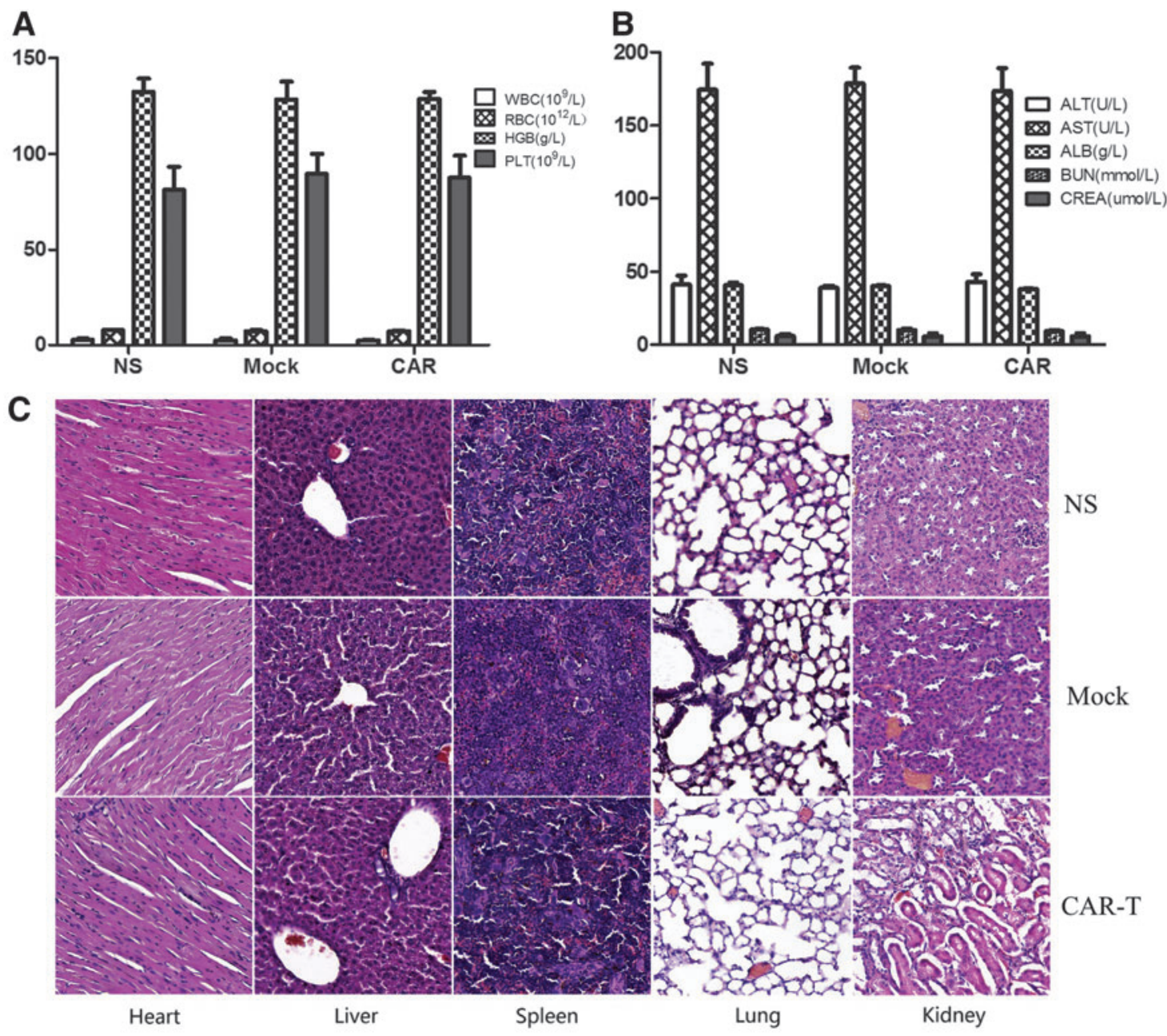

Figure 7. Systemic toxicity evaluation of CAR-modified T cells in the SW480 tumor model. (A) Routine blood test results. (B) Blood biochemical test results. (C) H\&E staining of visceral tissues, including the heart, liver, spleen, lung, and kidney in the SW480 model.

moting cell proliferation, survival, and metastasis. $^{2,3}$ In addition, EpCAM has been identified as a biomarker for CTCs and CSCs., ${ }^{9,14,16}$ Therefore, EpCAM may be an even more interesting target for cancer immunotherapy. Using flow cytometry, this study independently validated EpCAM protein expression in several solid cell lines and found that EpCAM is widely expressed in a broad panel of human tumor cells (Fig. 3A).

$\mathrm{mAs}$ against EpCAM are now in clinical trials for treating various cancers but do not lead to a cure, ${ }^{20,21,23,24}$ which may be attributed to low affinity to the target antigen, as well as some other ambiguous factors. This study proposed improving the therapeutic benefits by generating a CAR that targets EpCAM. For CAR-T cell therapy, the cytotoxicity and survival of the modified T cells are very vital for antitumor efficacy. Numerous studies have reported that costimulatory factors such as CD28, 4-1BB (CD137), CD27, and OX-40 can augment the effects of the cytokine release and enhance T-cell proliferation and persistence, which consequently improved antitumor potency. $^{26-28,32,33}$ Moreover, a large number of research studies have shown that the combination of CD28 and 4-1BB can enhance effector functions of CAR-T cells such as cytotoxicity, cytokine production, and persistence in vivo. ${ }^{27,32,34-37}$ To strengthen the antitumor effect of CAR-T in treating solid tumors further, this study incorporated the costimulatory factors CD28 and 4-1BB into the CAR (Fig. 1A and $\mathrm{B}$ ).

Currently, the most popular strategy used in the transfection of T lymphocytes is the viral vector, which is an efficient tool for the genomic integration of targeted genes. This study used the lentiviral vector for transduction and achieved efficient transfection efficiency. T-cell viability could 
not be affected by the lentivirus, and the transfection efficiency of lymphocytes was 25-35\% (Fig. 2A and B). CAR-T cells can be effectively amplified by about $>100$ times in 2 weeks. Although patients can be infused once with up to $10^{8}-10^{9}$ lymphocytes, these CAR-T cells will gradually reduce over time. Normal memory $\mathrm{T}$ cells can survive for several months to many years in the body and show a strong and effective immune response to target the tumor again after being stimulated by the associated antigens. It has been reported that $\mathrm{T}_{\mathrm{CM}}$ is the strongest of the tumor-specific effector cells, playing a main role in antitumor activity, and may be the most appropriate cell type for adoptive cell therapy. ${ }^{38-40}$ The present data indicate that $\mathrm{T}_{\mathrm{CM}}$ constitute a major part of CAR-T cells $(87.3 \%)$ after amplification (Fig. 2C).

The most critical contribution of this work is the demonstration of the applicability of EpCAMspecific CAR-T cells to target not only prostate cancer, ${ }^{41}$ but also other solid tumors such as breast carcinoma, NSCLC, and colon cancer. First, this study demonstrated that human T cells expressing EpCAM-specific CAR lysed a panel of $\mathrm{EpCAM}^{+}$cancer cells with high efficiency in vitro (Fig. 4). Furthermore, tumor cell killing and anticancer killing activity of CAR-T cells also resides in cytokine production. A significant IFN- $\gamma$ and TNF- $\alpha$ release by EpCAM-specific CAR-T cells was found over $20 \mathrm{~h}$ co-cultures with $\mathrm{EpCAM}^{+}$tumor cells (Fig. 3B and C). To evaluate EpCAM-specific CAR-T cell potential in vivo, two colon tumor models, HT29 and SW480, with high expression levels of EpCAM were adopted. EpCAM-specific CAR-T cells demonstrated powerful antitumor activity in vivo, with a dramatic inhibition of tumor growth in subcutaneous xenograft models (Fig. 5). Future studies will validate the ability of EpCAM-specific CAR-T cells to resist other solid tumors in vivo.

Unwanted toxicity is one of the major problems that limit CAR-T cell-based immunotherapy. The most common side effects of CAR-T cell therapies mainly depend on the expression levels of the tumor target in normal tissues. Currently, most of the tumor target antigens recognized by CAR-T cells are also expressed by healthy cells. Like other tumor target antigens, EpCAM is also expressed in many epithelial tissues as a cell adhesion molecule, and T lymphocytes redirected against EpCAM may lead to toxicity. However, the most toxic effects in mice were evaluated in preclinical experiments in this study, and CAR-T cell therapy did not induce any obvious systemic toxic effects. Even so, the clinical translation of this approach may thus benefit from the inclusion of a suicide gene within the CAR, which enables the CAR-modified T cells to be rapidly eliminated when undesired toxicity is observed.

In conclusion, the EpCAM-specific CAR was successfully constructed, which can be transduced into $\mathrm{T}$ cells and expressed steadily. CAR-T cells can proliferate quickly, mostly $\mathrm{CD}^{+}$and $\mathrm{T}_{\mathrm{CM}}$. $\mathrm{T}$ cells expressing EpCAM-specific CAR specifically recognize $\mathrm{EpCAM}^{+}$tumor cell lines, secrete high-dose IFN- $\gamma$ and TNF- $\alpha$ in vitro, and also significantly inhibit the formation and growth of various tumors in vivo. This study confirmed the antitumor ability of CAR-T cells targeting EpCAM and provided a new target for CAR-T cell therapies in treating solid tumors.

\section{ACKNOWLEDGMENTS}

This work is supported by the National Key Research and Development Program of China (2016YFC1303403), the National Natural and Scientific Foundation of China (81572981/H1611, 81672397/H1617, and 81703057/ H1611), the National High-tech R\&D program (863 Program; 2014AA020704), and the Key Scientific and Technological Foundation in Sichuan Province (17ZDZX0037). Parts of this work were used as a poster (PO-205) at the 17th Congress of Gastroenterology China 2017 Chinese Congress of Digestive Disease.

\section{AUTHOR DISCLOSURE}

Y-Q.W., Y-S.W., W.W., and B-L.Z. have submitted a patent concerning the methodology and application. Y-Q.W. and W.W. are scientific cofounders of Cygenpeutics and CarEne and hold the equity of the company. No conflicts of interests exist of the remaining authors.

\section{SUPPLEMENTARY MATERIAL}

Supplementary Figure S1 


\section{REFERENCES}

1. Herlyn M, Steplewski Z, Herlyn D, et al. Colorectal carcinoma-specific antigen: detection by means of monoclonal antibodies. Proc Natl Acad Sci U S A 1979;76:1438-1442.

2. Went PT, Lugli A, Meier $S$, et al. Frequent EpCam protein expression in human carcinomas. Hum Pathol 2004;35:122-128.

3. Nubel T, Preobraschenski J, Tuncay H, et al. Claudin7 regulates EpCAM-mediated functions in tumor progression. Mol Cancer Res 2009;7:285-299.

4. Brunner A, Prelog M, Verdorfer I, et al. EpCAM is predominantly expressed in high grade and advanced stage urothelial carcinoma of the bladder. J Clin Pathol 2008;61:307-310.

5. Fong D, Steurer M, Obrist $P$, et al. Ep-CAM expression in pancreatic and ampullary carcinomas: frequency and prognostic relevance. J Clin Patho 2008:61:31-35.

6. Spizzo G, Went P, Dirnhofer S, et al. High Ep-CAM expression is associated with poor prognosis in node-positive breast cancer. Breast Cancer Res Treat 2004;86:207-213.

7. Varga $M$, Obrist $P$, Schneeberger $S$, et al. Overexpression of epithelial cell adhesion molecule antigen in gallbladder carcinoma is an independent marker for poor survival. Clin Cancer Res 2004:10:3131-3136

8. Joosse SA, Gorges TM, Pantel K. Biology, detection, and clinical implications of circulating tumor cells. EMBO Mol Med 2015;7:1-11.

9. Schulze K, Gasch C, Staufer K, et al. Presence of EpCAM-positive circulating tumor cells as biomarker for systemic disease strongly correlates to survival in patients with hepatocellular carcinoma. Int J Cancer 2013;133:2165-2171.

10. Weissenstein U, Schumann A, Reif M, et al. Detection of circulating tumor cells in blood of metastatic breast cancer patients using a combination of cytokeratin and EpCAM antibodies. BMC Cancer 2012;12:206.

11. Coumans FA, Doggen CJ, Attard G, et al. All circulating EpCAM+CK+CD45- objects predict overall survival in castration-resistant prostate cancer. Ann Oncol 2010;21:1851-1857.

12. Khan MS, Tsigani T, Rashid M, et al. Circulating tumor cells and EpCAM expression in neuroendocrine tumors. Clin Cancer Res 2011;17:337-345

13. Al-Hajj M, Wicha MS, Benito-Hernandez A, et al Prospective identification of tumorigenic breast cancer cells. Proc Natl Acad Sci U S A 2003;100: 3983-3988.

14. Gires 0, Klein CA, Baeuerle PA. On the abundance of EpCAM on cancer stem cells. Nat Rev Cancer 2009;9:143; author reply 143.

15. Li C, Heidt DG, Dalerba P, et al. Identification of pancreatic cancer stem cells. Cancer Res 2007;67: 1030-1037

16. O'Brien CA, Pollett A, Gallinger $S$, et al. A human colon cancer cell capable of initiating tumour growth in immunodeficient mice. Nature 2007; 445:106-110.

17. Visvader JE, Lindeman GJ. Cancer stem cells in solid tumours: accumulating evidence and unresolved questions. Nat Rev Cancer 2008;8:755-768.

18. Riethmuller G, Holz E, Schlimok G, et al. Monoclonal antibody therapy for resected Dukes' $C$ colorectal cancer: seven-year outcome of a multicenter randomized trial. J Clin Oncol 1998;16: 1788-1794.

19. Punt CJ, Nagy A, Douillard JY, et al. Edrecolomab alone or in combination with fluorouracil and folinic acid in the adjuvant treatment of stage III colon cancer: a randomised study. Lancet 2002; 360:671-677.

20. Fields AL, Keller A, Schwartzberg L, et al. Adjuvant therapy with the monoclonal antibody Edrecolomab plus fluorouracil-based therapy does not improve overall survival of patients with stage III colon cancer. J Clin Oncol 2009;27:1941-1947.

21. Marschner N, Ruttinger D, Zugmaier G, et al. Phase II study of the human anti-epithelial cell adhesion molecule antibody adecatumumab in prostate cancer patients with increasing serum levels of prostate-specific antigen after radical prostatectomy. Urol Int 2010;85:386-395.

22. Xiang W, Wimberger $P$, Dreier T, et al. Cytotoxic activity of novel human monoclonal antibody MT201 against primary ovarian tumor cells. J Cancer Res Clin Oncol 2003;129:341-348.

23. Schmidt M, Ruttinger D, Sebastian M, et al. Phase IB study of the EpCAM antibody adecatumumab combined with docetaxel in patients with EpCAMpositive relapsed or refractory advanced-stage breast cancer. Ann Oncol 2012;23:2306-2313.

24. Eskander RN, Tewari KS. Epithelial cell-adhesion molecule-directed trifunctional antibody immunotherapy for symptom management of advanced ovarian cancer. Clin Pharmacol 2013;5:55-61.

25. Serrano LM, Pfeiffer T, Olivares $S$, et al. Differentiation of naive cord-blood T cells into CD19specific cytolytic effectors for posttransplantation adoptive immunotherapy. Blood 2006;107:26432652

26. Kowolik CM, Topp MS, Gonzalez S, et al. CD28 costimulation provided through a CD19-specific chimeric antigen receptor enhances in vivo persistence and antitumor efficacy of adoptively transferred T cells. Cancer Res 2006;66:1099511004

27. Milone MC, Fish JD, Carpenito C, et al. Chimeric receptors containing CD137 signal transduction domains mediate enhanced survival of T cells and increased antileukemic efficacy in vivo. Mol Ther 2009;17:1453-1464.

28. Song DG, Ye 0 , Carpenito $C$, et al. In vivo persistence, tumor localization, and antitumor activity of CAR-engineered T cells is enhanced by costimulatory signaling through CD137 (4-1BB). Cancer Res 2011;71:4617-4627.
29. Sallusto F, Lenig D, Forster R, et al. Two subsets of memory $T$ lymphocytes with distinct homing potentials and effector functions. Nature 1999; 401:708-712.

30. Sallusto F, Geginat J, Lanzavecchia A. Central memory and effector memory $T$ cell subsets: function, generation, and maintenance. Annu Rev Immunol 2004;22:745-763.

31. Gill S, June CH. Going viral: chimeric antigen receptor T-cell therapy for hematological malignancies. Immunol Rev 2015;263:68-89.

32. Carpenito C, Milone MC, Hassan R, et al. Control of large, established tumor xenografts with genetically retargeted human $T$ cells containing CD28 and CD137 domains. Proc Natl Acad Sci U S A 2009:106:3360-3365.

33. Zhong XS, Matsushita M, Plotkin J, et al. Chimeric antigen receptors combining $4-1 \mathrm{BB}$ and $\mathrm{CD} 28$ signaling domains augment PI3kinase/AKT/Bcl-XL activation and $\mathrm{CD} 8+\mathrm{T}$ cell-mediated tumor eradication. Mol Ther 2010:18:413-420.

34. Till BG, Jensen MC, Wang J, et al. CD20-specific adoptive immunotherapy for lymphoma using a chimeric antigen receptor with both CD28 and 4 $1 \mathrm{BB}$ domains: pilot clinical trial results. Blood 2012;119:3940-3950

35. Tammana S, Huang X, Wong M, et al. 4-1BB and CD28 signaling plays a synergistic role in redirecting umbilical cord blood T cells against Bcell malignancies. Hum Gene Ther 2010;21:75-86.

36. Karlsson H, Svensson E, Gigg C, et al. Evaluation of intracellular signaling downstream chimeric antigen receptors. PLoS One 2015;10:e0144787.

37. Heczey A, Liu D, Tian G, et al. Invariant NKT cells with chimeric antigen receptor provide a novel platform for safe and effective cancer immunotherapy. Blood 2014;124:2824-2833.

38. Yang S, Gattinoni L, Liu F, et al. In vitro generated anti-tumor T lymphocytes exhibit distinct subsets mimicking in vivo antigen-experienced cells Cancer Immunol Immunother 2011:60:739-749.

39. Klebanoff CA, Gattinoni L, Torabi-Parizi P, et al. Central memory self/tumor-reactive CD8+ T cells confer superior antitumor immunity compared with effector memory T cells. Proc Natl Acad Sci U S A 2005;102:9571-9576.

40. Berger C, Jensen MC, Lansdorp PM, et al Adoptive transfer of effector CD8+ $T$ cells derived from central memory cells establishes persistent $\mathrm{T}$ cell memory in primates. J Clin Invest 2008;118. 294-305.

41. Deng Z, Wu Y, Ma W, et al. Adoptive T-cell therapy of prostate cancer targeting the cancer stem cell antigen EpCAM. BMC Immunol 2015;16:1

Received for publication November 7, 2018; accepted after revision January 22, 2019

Published online: January 28, 2019 